\title{
WILEY-VCH
}

DOI: $10.1002 /(($ please add manuscript number $))$

Article type: Communication

\section{Wearable textile power module based on flexible ferroelectret and supercapacitor}

Sheng Yong ${ }^{1}$, Junjie Shi ${ }^{1}$, Stephen Beeby ${ }^{1 *}$

Dr S. Yong, Dr J. Shi, Prof. S. Beeby

Smart Electronic Materials \& System Research Group, School of Electronics and Computer

Science, University of Southampton, Southampton, SO17 1BJ, UK

E-mail: spb@ soton.ac.uk

Keywords: Energy power module, energy harvesting ferroelectret material, textile supercapacitor

\section{Abstract}

The rapid development of electronic textiles imposes a challenge on the power supply devices that, unlike conventional rigid batteries, ideally should be compatible with the mechanical properties. It would be highly advantageous if the power supply was integrated within the same textile as the system it is powering. This communication presents for the first time a textile power module that combines a ferroelectret biomechanical energy harvester and solid-state supercapacitor energy storage fabricated in a single woven cotton textile layer. The textile power module is highly flexible and the fluorinated ethylene propylene (FEP) based ferroelectret can generate electric energy with an instantaneous output voltage of $\sim 10 \mathrm{~V}$ and

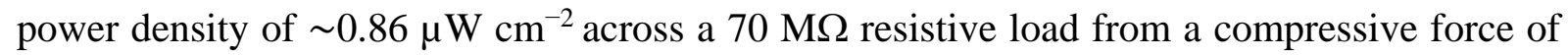
$350 \mathrm{~N}$. The activated carbon and non-toxic gel electrolyte based solid-state supercapacitor demonstrates a capacitance of $5.55 \mathrm{mF} \mathrm{cm}^{-2}$ and excellent stability after mechanically straining the textile. The textile power module can be charged to around $0.45 \mathrm{~V}$ in $3600 \mathrm{~s}$ with a compressive cyclical force of $350 \mathrm{~N}$ applied at $1 \mathrm{~Hz}$. This work demonstrates a promising 


\section{WILEY-VCH}

combination of materials and devices for achieving a self contained integrated power supply for e-textile applications.

Technologies for implementing wearable electronic devices are developing rapidly with increasing focus on the requirements of multifunctionality, flexibility and reliability. One such method of implementation is textile electronics (e-textiles, or smart fabrics). ${ }^{[1,2]}$ These systems require an efficient, flexible and durable power supply module, and meeting these demands is one of the biggest technical challenges for wearable electronics. At present, e-textiles systems are typically powered by conventional energy storage devices such as batteries that are neither flexible and require frequent charging. Traditional batteries alter the properties (e.g. breathability) and feel (e.g. shear and drape) of textiles and are not well suited to e-textile applications. Therefore combining a flexible energy harvester with a flexible energy storage supercapacitor in the same piece of textile could be an attractive solution for powering wearable e-textile devices.

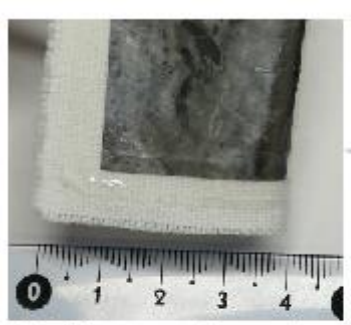

Textile energy harvester (ferroelectret)
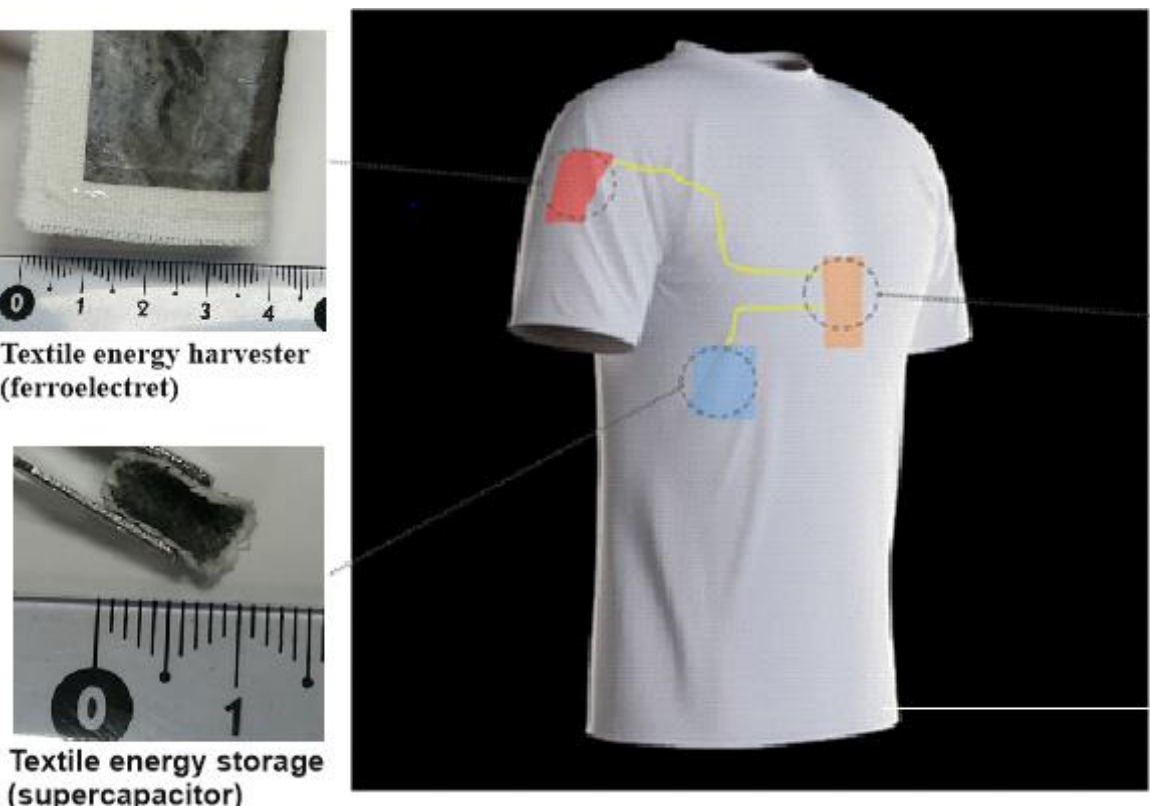

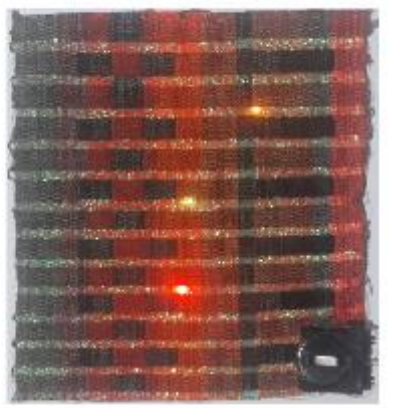

Textile electronics 


\section{WILEY-VCH}

Figure 1. Schematic of the textile power module comprising a spray deposited supercapacitor, ferroelectret energy harvester and electronics all integrated into the same single layer of textile. The textile electronics represents the power conditioning circuit and the wearable load electronics.

Figure 1 illustrates the concept of a flexible and scalable textile power module that can be fully integrated in the same piece of fabric. It consists of a ferroelectret energy harvester for capturing energy from mechanical movement, a power management circuit to convert the electrical energy into a form suitable for storing and a supercapacitor providing an energy reservoir. The textile power module can be used to supply the load electronics that can be incorporated in the same fabric. The module provides a flexible energy storage solution that both buffers the intermittent output typical of energy harvesting power supplies and overcomes the limited energy storage capacity available without separate recharging. This textile power module is the first example of a storage device, harvester and circuit being realised in the same piece of standard fabric. The materials used and structures developed can be used in most standard textiles and can be fabricated using low cost processes such as printing, spray coating and laminating.

Supercapacitors are an electrochemical energy storage device that function via two types of capacitive mechanisms: the electrical double layer capacitance (EDLC) and redox pseudocapacitance. ${ }^{[3]}$ Supercapacitors offer a high power density, excellent cyclical stability, safe operation and can be easily integrated into textiles using 1D supercapacitor yarns or carbon impregnation techniques. ${ }^{[4]}$ Yarn based supercapacitors that can be woven into a textile have been successfully demonstrated with different configuration and electrode materials. For example, fiber electrodes made from carbon nanotube, ${ }^{[5]}$ activated carbon fiber, ${ }^{[6]}$ reduced graphene oxide ${ }^{[7]}$ and redox materials such as Manganese Oxide. ${ }^{[8]}$ Textile supercapacitors can also be realised by coating the fabric with carbonaceous materials that can form conductive 


\section{WILEY-VCH}

electrodes. Textiles provide a porous substrate that is well suited for use as a scaffold structure for creating the supercapacitor electrodes. Furthermore, despite its flexibility, most woven textiles that do not contain elastane fibers will not stretch along the warp direction and exhibit only minimal stretch in the weft direction. This means woven fabrics are mechanically stable and are well suited to the formation of stable electrodes. Knitted fabrics, on the other hand, are inherently stretchable and forming consistent conductive networks on such substrates will be problematic. Previous work has demonstrated textile supercapacitors realised by forming a multilayer structure by stacking different fabric layers. ${ }^{[9]}$ The two textile electrodes have previously been fabricated separately on different pieces of fabric and are then placed together with a layer of dried gel electrolyte or a polymer separator layer positioned in between. The multilayer textile supercapacitor design increases the fabrication complexity since it requires the different layers to be reliably bonded together and the finished assembly will be less flexible than a normal fabric. With respect to practical applications in clothing, most garments are formed from a single textile layer and stacking textile layers could be problematic since it will alter the thickness and the thermally insulating properties of the textile potentially making it uncomfortable for the user. We have previously demonstrated a solid-state supercapacitor implemented in a single woven cotton textile layer ${ }^{[10]}$. An activated carbon and carbon black solution was spray coated in a highly controlled manner on both sides of the cotton textile. By precisely controlling the distance, speed and pneumatic pressure of the spray coating process, the amount of carbon deposited and the distance it soaks into the cotton can be regulated. This leaves carbon electrodes on both sides of the fabric separated by an uncoated region at the center of the cotton textile that acts as the separator in the supercapacitor and, given the porous nature of the cotton fibers, can absorb the gel electrolyte. The single textile layer device achieved a specific area capacitance of $49.1 \mathrm{mF} \mathrm{cm}^{-2}$, excellent cycling stability demonstrating less than a $1 \%$ change in capacitance after 15000 charge/discharge cycles and good mechanical durability. ${ }^{[\text {Error! Bookmark not defined.] }}$ The work presented here builds upon these results. 


\section{WILEY-VCH}

Triboelectric nano generators (TENGs) have been used to realise textile based mechanical energy harvesters and these have been combined with supercapacitors. Initially, fiber based TENGs were electrically connected to a separate yarn supercapacitor ${ }^{[11]}$ but more recently these have been combined into a textile using a variety of approaches and materials. ${ }^{[12,13,14,15]}$ These examples demonstrate the feasibility of a textile power module with power levels of $10 \mu \mathrm{W} \mathrm{cm}{ }^{-2}$ and stored energies of $1.6 \mu \mathrm{Wh} \mathrm{cm}^{-2}$ having been achieved. An alternative to TENG's is to use ferroelectret materials (also known as piezoelectrets), typically fabricated from cellular polymers such as porous polypropylene, are internally charged and behave like soft and sensitive piezoelectric materials that can be used to transduce mechanical forces into electrical signals and vice versa. ${ }^{[16]}$ During polarization, the voids inside the ferroelectret become charged with positive and negative charges being trapped on the top and bottom surfaces of the voids. A macroscopic dipole moment is formed by these separate charged voids resulting in net piezoelectric like properties. The soft, flexible nature of the polymer ferroelectret makes it highly suitable for use in human applications and also yields high $d_{33}$ values compared with its polymer piezoelectric counterparts such as polyvinylidene fluoride (PVDF). These properties make ferroelectret materials attractive for energy harvesting from human motion. Since the quasi-piezoelectric effect is related to the geometry change in the charged voids, materials and assemblies other than the typical cellular foams have been demonstrated. For example, voids can be specifically microengineered in polydimethylsiloxane $(\mathrm{PDMS})^{[17]}$ films and performance can be improved by optimising void geometry. ${ }^{[18]}$ Porous poly vinylidenefluoride-co-hexafluoropropylene ${ }^{[} \quad 19 \quad$ ], polypropylene $^{[} \quad 20$ ], and polytetrafluoroethylene ${ }^{[21]}$ are alternative electret materials used in ferroelectret generators. These devices have demonstrated a peak power of $0.69 \mu \mathrm{W} . \mathrm{cm}^{-2}, 0.85 \mu \mathrm{W} . \mathrm{cm}^{-2}$ and 0.73 $\mu \mathrm{W} . \mathrm{cm}^{-2}$ respectively. The work presented here is based upon previously published results presented by the authors forming a ferroelectret from two fluorinated ethylene propylene (FEP) sheets separated by a standard polymer foam forming a void between the sheets. ${ }^{[2]}$ The charge 


\section{WILEY-VCH}

is trapped on the surface of the FEP sheet and the polymer foam acts as a mechanical spacer and provides the restoring force that returns the voids to their original geometry. The flexile FEP-polymer foam ferroelectret demonstrated a power density of over $6.4 \mu \mathrm{W} \mathrm{cm}{ }^{-2}$ with a resistive load of $10 \mathrm{M} \Omega .^{[17]}$

In this paper, we report a textile power module fabricated in a single standard cotton layer using a combination of standard processes commonly employed by the textile industry such as screen printing and spray coating. The materials are low-cost and safe to use resulting in a scalable solution that can be applied to a wide range of standard fabrics. This work uses a textile based ferroelectret formed from an amalgamation of FEP and cotton. The textile forms an integral part of the harvester acting as the spacer material as shown in Figure 2a. After corona poling, the trapped charge on the surface of the FEP film is shown in Figure $2 \mathrm{~b}$ and when the textile is deformed by, for example, a compressive force and, in order to maintain electrical neutrality, the change in the electrical field across the void is compensated by a variation in the induced charge on the electrodes resulting in a net current flow. The FEP textile ferroelectret did not exhibit any triboelectric effect. The triboelectric effect arises when two dielectric materials from opposite ends of the triboelectric series come into contact. Since three layers are used in a symmetrical structure with two FEP/cotton interfaces, any charge arising from the triboelectric effect at these interfaces cancels out. This confirms the unpolarised ferroelectret devices did not generate electrical output. 


\section{WILEY-VCH}

a

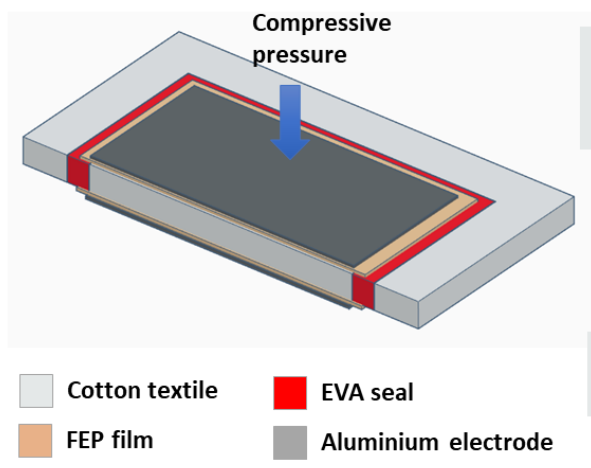

b

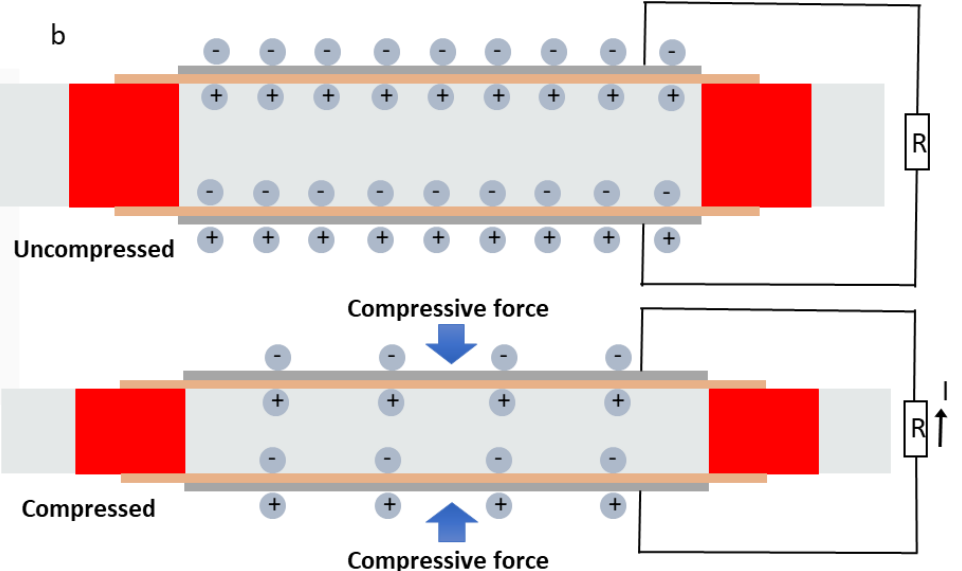

Figure 2. The textile ferroelectret. a) Schematic of the ferroelectret structure showing the fabric sandwiched between the FEP films with the thin film aluminum electrodes and EVA seal; b) The operating principle of the ferroelectret showing the flow or charge when the textile is compressed.

This is combined with a fully solid-state supercapacitor formed from carbon electrodes (activated carbon and carbon black) spray coated on to both sides of the textile and impregnated with an ammonium dihydrogen phosphate (ADP)/Polyvinyl-alcohol (PVA) electrolyte. The harvester and storage elements are fabricated at separate locations on the same piece of textile and are electrically connected by a screen printed flexible circuit with an integrated diode rectifier mounted on the fabric. This paper demonstrates the successful integration of all the key elements of a textile power supply module on a single standard fabric substrate paving the way for autonomous wearable e-textile devices. 


\section{WILEY-VCH}
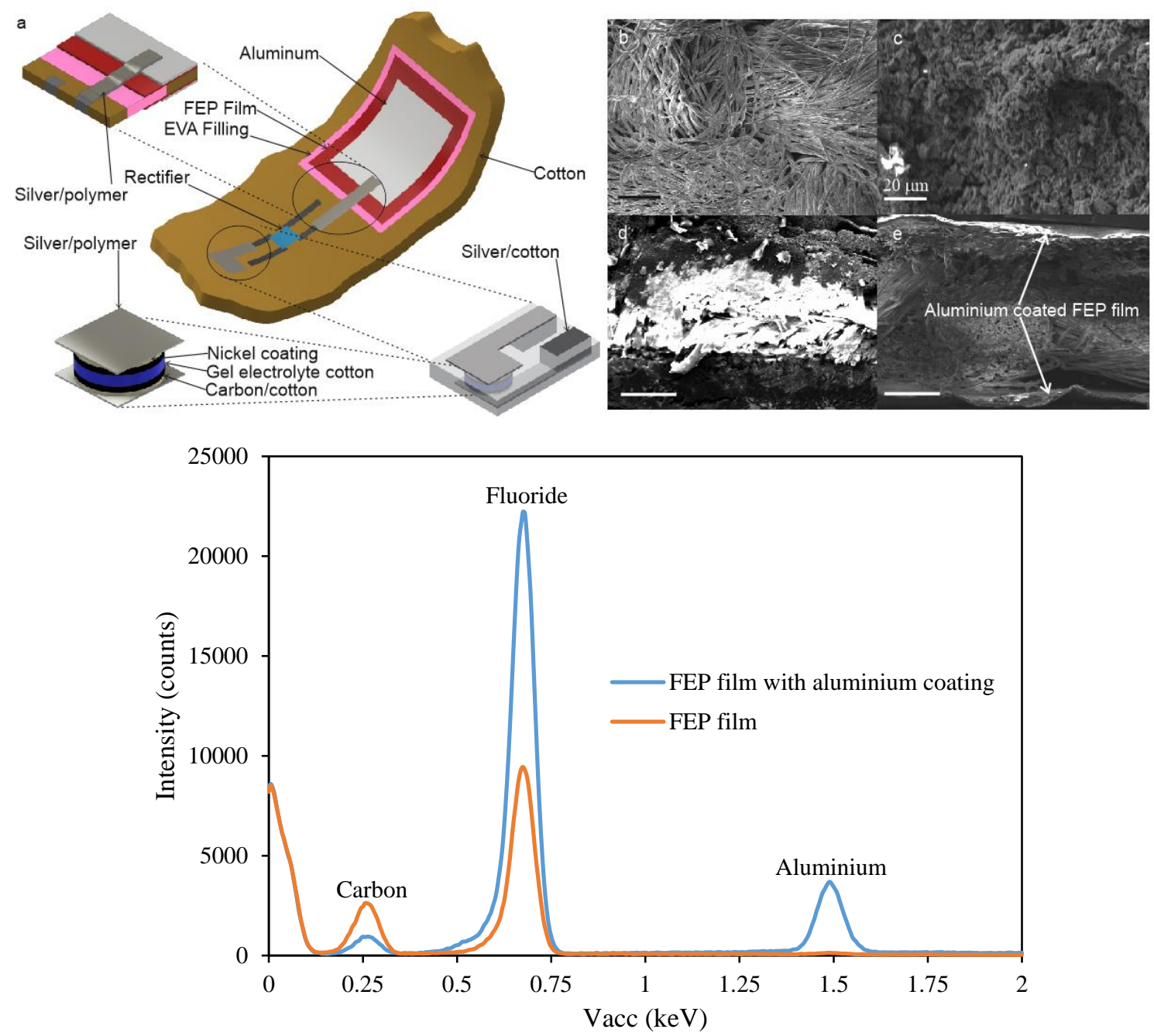

Figure 3. The textile power module. a) Schematic of the textile power module showing the cross-sections of the supercapacitor and ferroelectret components and through textile silver contacts. Scanning electron microscopy (SEM) images of b) the uncoated cotton fibers (plan view); c) the carbon coated cotton fibers (plan view); d) cross-section of the supercapacitor with the silver polymer current collector, e) cross-section of the ferroelectret. The scale bars are 200 $\mu \mathrm{m}$ unless otherwise shown, f) EDX results of the FEP films with and without aluminum

The schematic of one side of the textile power module is shown in Figure 3a and the cross-sections show the structure of the key components. The textile appears identical on the reverse side but without the rectifier. The design requires electrical connections between both sides of the textile in order to connect the ferroelectret electrodes to the supercapacitor 


\section{WILEY-VCH}

electrodes via the rectifier. These through textile connections are screen printed from both sides using standard silver polymer ink that soaks through the $500 \mu \mathrm{m}$ thick cotton fabric making electrical connections with each side. The structures of the FEP-cotton ferroelectret and supercapacitor were examined using a scanning electron microscope (SEM). The bare cotton fibers are shown in figure $3 \mathrm{~b}$, and after spraying the carbon coats the surface of the fabric and penetrates through the fibers (figure $3 \mathrm{c}$ ). A binder comprising $0.4 \%$ by weight of ethylene-vinyl acetate was added to the carbon spray solution to improve the adhesion of the carbon particles to the fiber and therefore the mechanical and electrochemical stability of the supercapacitor. Figure 3d shows the SEM image of the cross-sectional of the single layer cotton supercapacitor. The center of the image where the fibres are not covered by the carbon particles has been charged by the SEM and therefore appears much brighter in the photograph. The darker parts in the image indicate the depth of the carbon electrodes and a clear gap is visible forming a well-defined, stable charge separator within the textile. Figure 3e shows an SEM photograph of a cross section of the ferroelectret taken at its center. The SEM charged lighter areas at the top and bottom of the fabric indicate the edges of the two FEP films and the aluminum electrodes are evaporated on to the exposed top surface of each FEP film. Figure $3 \mathrm{f}$ shows the Energy dispersive X-ray spectroscopy (EDX) results of the FEP films with and without aluminium coating, The EDX results show the presence of carbon $(0.26 \mathrm{keV})$ and fluoride $(0.675 \mathrm{keV})$ in both samples. For FEP film with aluminium coating, a strong intensity (counts) peak appear at $1.49 \mathrm{keV}$, it shows the presence of aluminium electrode on top of FEP film.

The piezoelectric coefficient $d_{33}$ of the FEP textile ferroelectret measured after poling was $520 \mathrm{pC} . \mathrm{N}^{-1}$. In addition to being highly compressible and flexible, the textile also prevents the FEP films from touching that would otherwise discharge the trapped charge and render the ferroelectret inactive. After polarization, the charge trapped on the inside surface of the FEP films could leak away through the textile and therefore the ferroelectret must be sealed by an 


\section{WILEY-VCH}

ethylene-vinyl acetate (EVA) polymer annulus. This is spray coated from both sides of the fabric through a suitable mask and soaks into the fabric forming a stable hermetic barrier.

Figure 4a shows the output voltage from a FEP-textile ferroelectret under a $350 \mathrm{~N}$ cyclical compressive force applied at $1 \mathrm{~Hz}$. The negative peak output voltage is about $-13 \mathrm{~V}$ and this is generated from the application of the compressive force. The positive voltage of around $3 \mathrm{~V}$ is produced by the ferroelectret returning to its original thickness after the compressive force is released. The difference in magnitude of the positive and negative voltages is explained by the rate of application of the applied force (see equation 1 in the Experimental Section). For the negative peak, the time taken to ramp up from 0 to $350 \mathrm{~N}$ is controlled by the ElectroPuls E1000 test rig and was 0.1 seconds. In contrast, for the positive peak the ferroelectret cell takes around 0.5 seconds to return to its original geometry and hence the voltage is lower. The time taken is determined by the elastic properties of the cotton textile and the FEP films. The amount of energy generated is a function of the magnitude of the voltage and the duration of the output pulse. Therefore, the lower positive voltage peak is compensated by the increased pulse duration. From equation 2 in the experimental section, the calculated peak power output from the negative peak voltage is $16.9 \mu \mathrm{W}$, whilst for the positive and negative voltage segments the energy output is $0.13 \mu \mathrm{J}$ and $0.31 \mu \mathrm{J}$ respectively. Figure $4 \mathrm{~b}$ shows the output voltage of the FEP textile ferroelectret under different compressive forces. The peak voltage rises with the increasing force and saturates at round $-20 \mathrm{~V}$ for a compressive force of around $550 \mathrm{~N}$ at which point the textile spacer has been fully compressed. The relatively linear increase in voltage with applied force indicates the modulus of compression remains essentially constant up until saturation. The output peak power of the ferroelectret was investigated as a function of external load resitances, as shown in Figure 4c. The maximum peak power is $31 \mu \mathrm{W}\left(0.86 \mu \mathrm{W} . \mathrm{cm}^{-2}\right)$ achieved at a $70 \mathrm{M} \Omega$ resistive load. Finally, as shown in Figure $4 \mathrm{~d}$, the measured $d_{33}$ after 30 days was $480 \mathrm{pC} \mathrm{N}^{-1}$ indicating the trapped charge is stable. 
WILEY-VCH
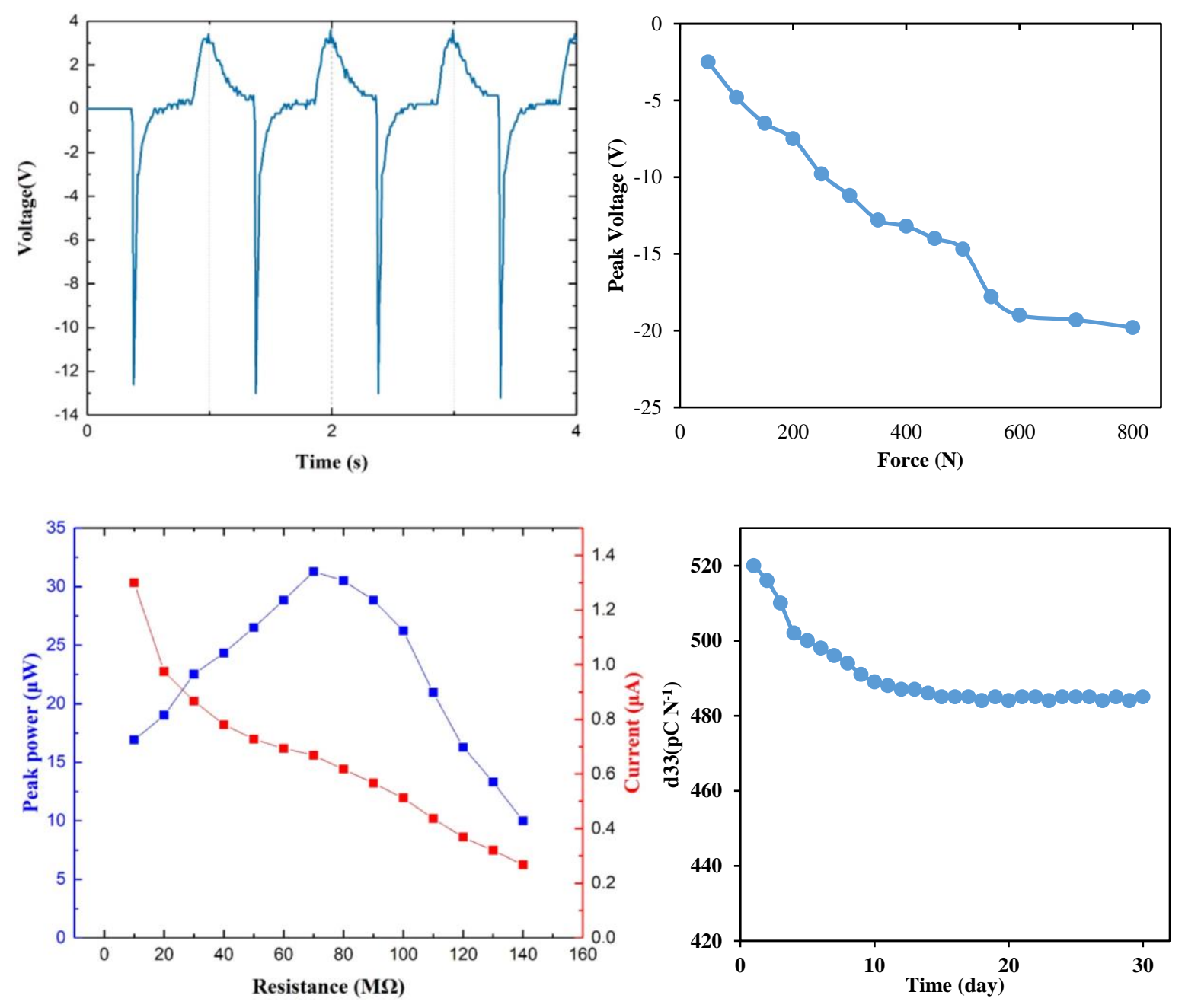

Figure 4. FEP-textile ferroelectret output. a) Output voltage of textile based FEP ferroelectret under cyclical $350 \mathrm{~N}$ compressive force with $10 \mathrm{M} \Omega$ load; b) Peak output voltage versus compressive force; c) Output power and current of the ferroelectret as a function of external load resistances; d) long term $d_{33}$ measurement

Following the ferroelectret fabrication, the area of cotton defined by the carbon electrodes was vacuum impregnated by a PVA/ $\mathrm{NH}_{4} \mathrm{H}_{2} \mathrm{PO}_{4}$ gel electrolyte. Nickel-silver-polyurethane current collectors were then bonded to the carbon electrodes to complete the fabrication of the supercapacitor and make electrical connections to the screen printed silver tracks. The final assembly step is to encapsulate the textile power module using a screen printed waterproof polyurethane layer. This was applied across the entire module apart from on the aluminum 


\section{WILEY-VCH}

electrodes of the ferroelectret, which were left uncoated minimize the compressive modulus and therefore maximise the $d_{33}$ coefficient. The encapsulation layer was cured under a compressive load to improve adhesion and reduce contact impedances by compressing the assembled device. The electrochemical performance of the encapsulated single layer cotton supercapacitor was evaluated with a symmetrical two-electrode configuration with no mechanical pressure applied. The cyclic voltammetry (CV) results shown in figure 5a from scan rates of 50 to $200 \mathrm{mV} \cdot \mathrm{s}^{-1}$ exhibit the normal rectangular shape but is inclined due to the contact impedance between the current collector and supercapacitor electrodes. Compressive pressure reduces this contact impedance resulting in a less inclined rectangular shape even at higher scan rates. Figure 5a also indicates the textile supercapacitor is electrochemically stable and without obvious redox reaction. The capacitance calculated from the $25 \mathrm{mV} \cdot \mathrm{s}^{-1}$ curve is 23 $\mathrm{mF} . \mathrm{cm}^{-2}$ and this drops by $43 \%$ when the $\mathrm{CV}$ test scan rate increases to $200 \mathrm{mV} . \mathrm{s}^{-1}$ as shown in Figure 5b. The textile supercapacitor was also galvanostatically charged/discharged (GCD test) at current densities from 1 to $5 \mathrm{~mA} \cdot \mathrm{cm}^{-2}$ (see Figure $5 \mathrm{c}$ ). The initial vertical IR drop and the sharp reduction in cycling time with increasing current density is due to the contact impedance. At $1 \mathrm{~mA} \cdot \mathrm{cm}^{-2}$, the textile supercapacitor demonstrates specific area and volume capacitances of $5.5 \mathrm{mF} . \mathrm{cm}^{-2} 101 \mathrm{mF} . \mathrm{cm}^{-3}$ but this declines with increasing cycling current as shown in see Figure $5 \mathrm{~d}$. This is due to the contact impedances within the device. The Ragone plot in the figure 5e was devised from GCD test between $1 \mathrm{~mA} . \mathrm{cm}^{-2}$ and $5 \mathrm{~mA} . \mathrm{cm}^{-2}$, it shows the area energy density of the textile supercapacitor increases from $0.01 \mu \mathrm{Wh} \mathrm{cm}^{-2}$ to $0.49 \mu \mathrm{Wh} \mathrm{cm} \mathrm{cm}^{-2}$ with area power density of least $0.5 \mathrm{~mW} . \mathrm{cm}^{-2}$ for all test current densities. Given the supercapacitor area of $0.36 \mathrm{~cm}^{2}$, the final capacitance base on the GCD results was $2 \mathrm{mF}$. In comparison with the same textile supercapacitor evaluated in a Swagelok PFA tube fitting, ${ }^{\text {[Error! }}$ Bookmark not defined.] the device presented in this paper demonstrated lower area capacitance, energy density and power density. This is because the previous supercapacitor was placed under 


\section{WILEY-VCH}

compression during testing in the tube fitting which reduces the thickness and resistance of the device resulting in an increased measured capacitance.
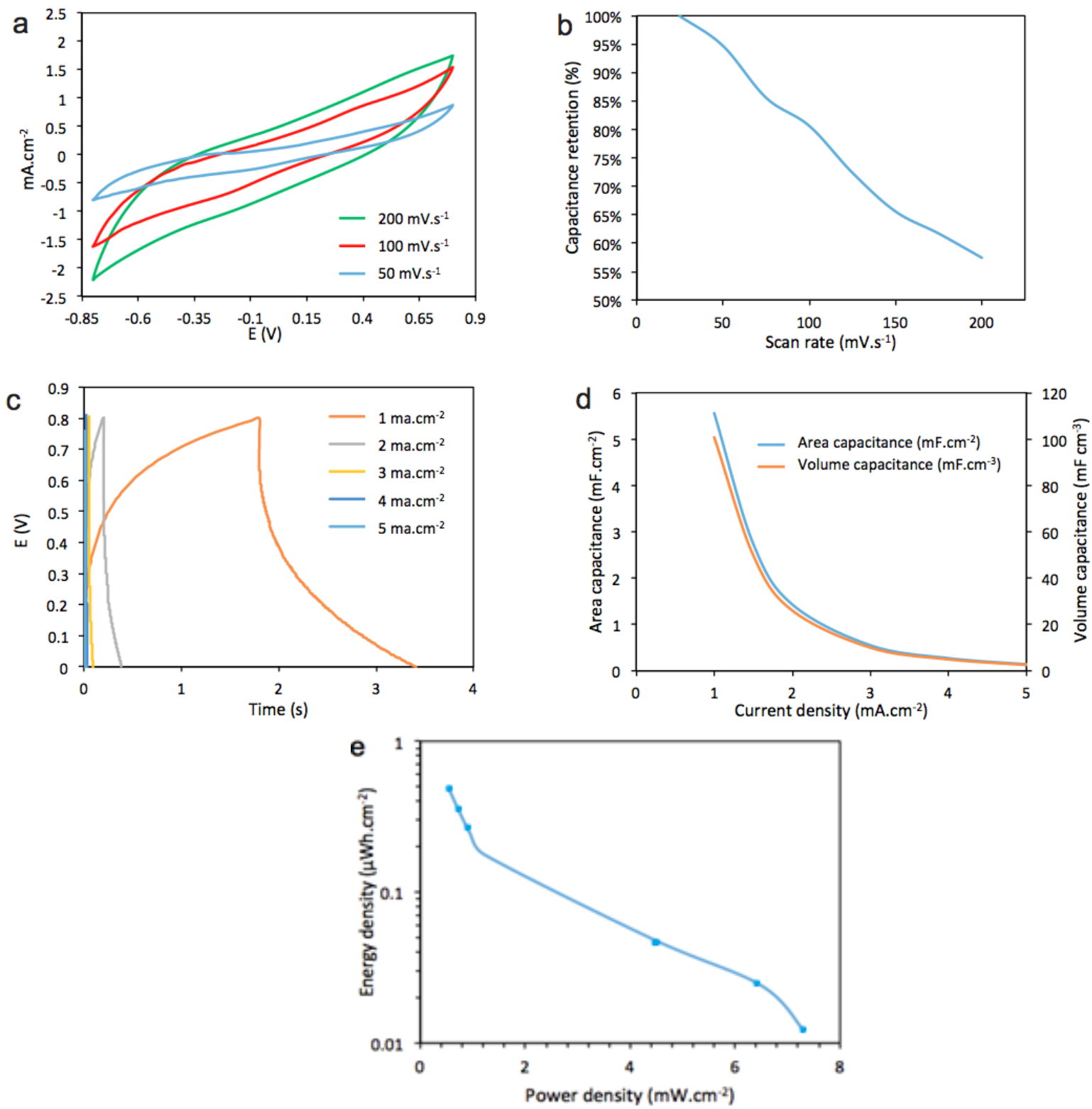

Figure 5. Electrochemical performance of the single layer cotton supercapacitor. a) CV test results between $+/-0.8 \mathrm{~V}$ at scan rates of $200,100,50 \mathrm{mV} \mathrm{s}^{-1}$; b) $\mathrm{CV}$ derived capacitance retention between $+/-0.8 \mathrm{~V}$ at the scan rates from 25 to $200 \mathrm{mV} \mathrm{s}^{-1}$; c) Galvanostatic charge/discharge test (GCD) of the textile supercapacitor, test current 1 to $5 \mathrm{~mA} \mathrm{~cm}^{-2}$ between 0 - $0.8 \mathrm{~V}$; d) GCD derived area and volume capacitance (test current 1 to $5 \mathrm{~mA} \mathrm{~cm}{ }^{-2}$ ) between 


\section{WILEY-VCH}

0 - 0.8 ; ;e) GCD derived Ragone plot at increasing current densities between 1 and $5 \mathrm{~mA} \mathrm{~cm}{ }^{-2}$ tested at $0.8 \mathrm{~V}$.

a
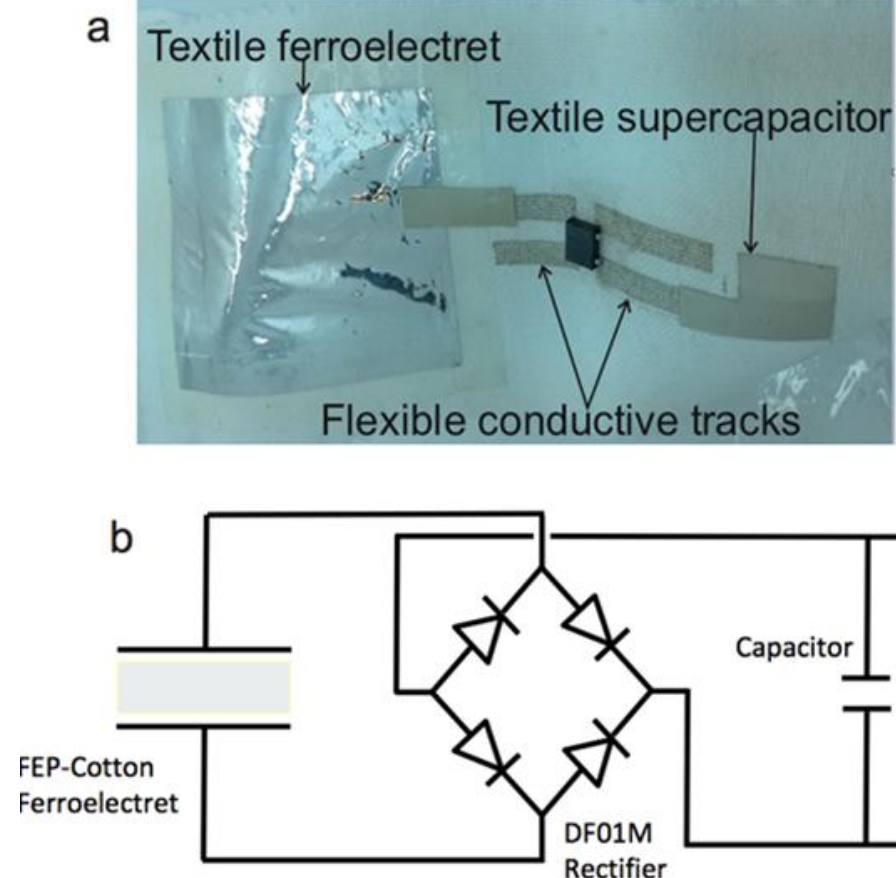

C

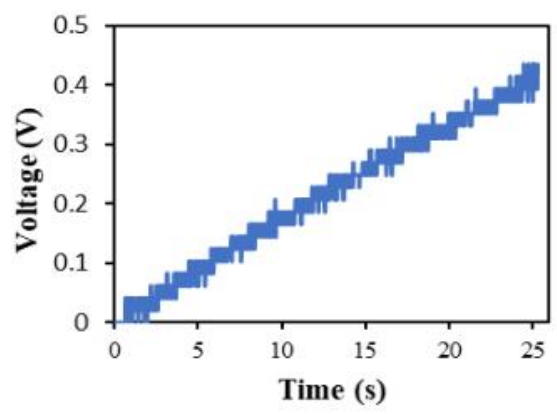

d

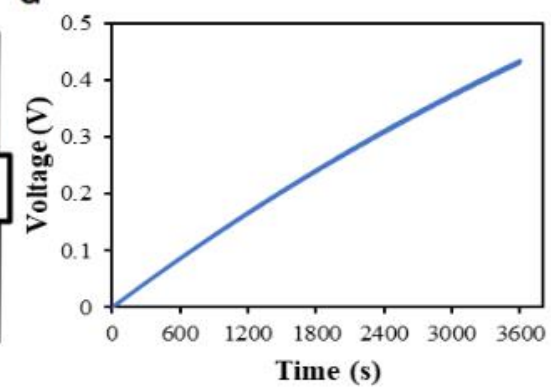

Figure 6. Textile power module performance. a) Assembled textile power module; b) Circuit diagram of the ferroelectret harvester, rectifier and energy storage; c) FEP-Textile ferroelectret charging an external $10 \mu \mathrm{F}$ capacitor; d) FEP-Textile ferroelectret charging the $2 \mathrm{mF}$ textile capacitor.

The final assembled textile power module is shown in Figure 6a. A correct measure of the practical energy harvesting potential of the module is the time taken to charge the storage capacitor. In order to this, the output voltage must be rectified and this was achieved using a commercial bridge rectifier packaged integrated circuit (DF01M) as shown in the equivalent circuit in Figure $6 \mathrm{~b}$. The ferroelectret was first tested connected to a separate $10 \mu \mathrm{F}$ capacitor and driven by a cyclical mechanical input of $350 \mathrm{~N}$ compressive force at $1 \mathrm{~Hz}$ intervals for 30 


\section{WILEY-VCH}

seconds. The voltage across the capacitor increases to $0.45 \mathrm{~V}$ as shown in Figure 6c. The energy delivered to the capacitor each cycle can be calculated with the total energy accumulated during the entire charging process of $1.01 \mu \mathrm{J}$ ( 25 cycles in total), corresponding to an average energy of $0.042 \mu \mathrm{J} /$ cycle. Under the same test conditions, the voltage across the textile super-capacitor increases to $0.45 \mathrm{~V}$ (3600 cycles in total) as shown in Figure 6d. Given the capacity of $2 \mathrm{mF}$ and the gel electrolyte that limits the voltage to $1.5 \mathrm{~V}$ [Error! Bookmark not defined.], it would take about 10 hours to fully charge the device assuming the same energy output per cycle. Mechanical fatigue resistance is also an important factor for the textile power module. The textile ferroelectret has been tested up to 21,000 compressive cycles at $350 \mathrm{~N}$ and shows deterioration in output. The textile supercapacitor has been cyclically bent 500 times around a diameter of $2.5 \mathrm{~cm}$ and the $\mathrm{CV}$ plots obtained before and after show a small reduction in capacitance of less than $2 \%$. This indicates that the electrochemical performance of the supercapacitor is not greatly affected by the application of repeated bending strains. However, because the circuit in the textile power module is a conventional rigid dual in line DFM package, its attachment to the textile is not suited to cyclical bending and the die does not withstand washing.

This work has demonstrated the feasibility of realizing a full textile power module on a single piece of standard fabric. The ferroelectret is capable of charging the textile supercapacitor via the rectifier circuit mounted on the same fabric. The complete integration on the same piece of fabric does introduce some compromises and integration challenges. This means the performance of the system is not yet as good as the individual components have previously demonstrated when fabricated and tested in isolation. One particular challenge that should be noted is the extra impedance that arises from the contact between the supercapacitors carbon electrodes and the nickel-silver-polyurethane current collectors and again between the current collectors and printed silver tracks. The materials, processes and structures demonstrated here 


\section{WILEY-VCH}

can be applied to most standard textiles and this work points the way to a practical solution to the challenge of powering wearable e-textile systems.

\section{Experimental section}

The following materials and processes were all applied to a standard cotton textile with a thickness of $500 \mu \mathrm{m}$

Fabrication of carbon electrodes: The carbon solution was prepared with activated carbon (YP80F is from Kuraray Chemical), conductive agent Shawinigan Black poly (ethylene-vinyl acetate) binder dissolved in 1,2,4-Trichlorobenzene solvent and a sodium dodecyl sulphate surfactant. The ratio of carbon to binder was $85 / 15$ by weight. The surface of the cotton textile was first shaved to reduce pilosity and the carbon solution was spray coated through a mask on both side of the textile substrates in a fume cabinet at room temperature. The spray nozzle (diameter of $0.2 \mathrm{~mm}$ ) was positioned $10 \mathrm{~cm}$ away from the fabric and an air pressure of $25 \mathrm{psi}$ (1.72 bars) used for the spraying. Each side of the textile sample was sprayed for 2 seconds. The mask was removed and the textile substrate was dried in a fan oven at $150^{\circ} \mathrm{C}$ for 10 minutes.

Fabrication of silver conductive tracks on cotton and supercapacitor currents collector film:

The silver conductive tracks were fabricated using a screen printed a silver polymer paste (DuPont d5000). The 4 silver tracks were $5 \mathrm{~mm}$ wide by $20 \mathrm{~mm}$ long and the paste was cured in a box oven for 10 minutes at $50^{\circ} \mathrm{C}$. The bridge rectifier IC was placed at the center of the silver tracks and the chip pins were punch through the fabric and bonded with silver epoxy to the printed silver tracks. The supercapacitor currents collector films were fabricated separately by screen printing silver and polyurethane layers on top of a PDMS substrate. The silver/polyurethane layers were thermally cured and peeled off the PDMS. The nickel film was then spray coated on top of the silver. The nickel ink was formed from nickel powder (average 


\section{WILEY-VCH}

size of $5 \mu \mathrm{m}$, from Sigma-Aldrich), Isoamyl acetate solvent, sodium dodecyl sulphate surfactant and $0.8 \%$ by weight of EVA binder material to improve adhesion.

Single cotton layer supercapacitor assembly: The gel electrolyte was prepared by mixing $0.3 \mathrm{~g}$ of ammonium dihydrogen phosphate (ADP) salt in $5 \mathrm{~mL}$ DI water with $0.5 \mathrm{~g}$ polyvinyl-alcohol (PVA) polymer and heated to $90^{\circ} \mathrm{C}$ under constant stirring clear. After cooling down to room temperature, the gel electrolyte was degassed under vacuum (0.029 psi / 20 mbar) for 2 hours. The cotton textile electrode was dipped into the degassed gel electrolyte under vacuum for 20 minutes and cured under compression for 1 hour at $40^{\circ} \mathrm{C}$. Finally two pieces of current collector films bonded to the fabric and the assemble was sealed by screen printing a UV curable polyurethane layer.

Preparation of FEP films: The FEP sheet (DuPont, $304 \mathrm{~mm} \times 200 \mathrm{~mm} \times 25 \mu \mathrm{m}$ ) were cut to a size of $70 \mathrm{~mm} \times 70 \mathrm{~mm}$. Aluminum electrodes were evaporated on to one side (Bak600 Evaporator, Levbold Ltd.) using a shadow mask to define an electrode area of $60 \mathrm{~mm} \times 60 \mathrm{~mm}$ with a thickness of $100 \mathrm{~nm}$.

Fabrication of FEP-cotton textile ferroelectret: EVA binder $(0.5 \mathrm{~g})$ was dissolved in 1,2,4Trichlorobenzene solvent $(10 \mathrm{~mL})$ and spray coated into the cotton textile around the ferroelectret area patterned by a metal mask to form the EVA annulus. This was cured for 2 hour at $80^{\circ} \mathrm{C}$ and the aluminum coated FEP films were aligned to the EVA annulus on both sides and bonded to the cotton textile by adhesive polymer film (Tesa ${ }^{\circledR} 64621$ double sided tape) with aluminum side up. Nickel-silver films were used to connect the aluminum to the printed silver tracks and the textile power module was sealed by a UV curable screen printed polyurethane layer. The ferroelectret was polarized in a corona poling rig with a corona-tip voltage of $-25 \mathrm{kV}$ with a charging time of 60 seconds. 


\section{WILEY-VCH}

Characterization of FEP-cotton textile ferroelectret: the $\mathrm{d}_{33}$ coefficient of the ferroelectrets were measured using a PiezoMeter (PM300, Piezotest Ltd). This system provides a direct reading of $d_{33}$ by clamping the sample in series with a reference sample and applying a known force to both. The electrical signal from the sample is compared to the built in reference enabling the $d_{33}$ value to be calculated. The generated output voltage across the resistive load R can be expressed as:

$$
V=\frac{\Delta Q}{t} R
$$

Where $V$ is the output voltage, $\Delta Q$ is the variation in charge and $t$ is the time taken to apply the force. The electrical energy generated can be calculated using equation 2 .

$$
U=\int_{t_{1}}^{t_{2}} \frac{V(t)^{2}}{R} d t
$$

Electrochemical testing of energy textile: Electrochemical tests were performed using a VMP2 potentiostat/galvanostat (Biologic, France). Electrochemical impedance spectroscopy (EIS) was carried out at frequencies varying from $20 \mathrm{mHz}$ to $20 \mathrm{k} \mathrm{Hz}$ with a peak to peak amplitude of $20 \mathrm{mV}$ in open circuit. Cyclic voltammetry (CV) results were collected at scan rates of 25 , 50, 100, 150 and $200 \mathrm{mV} . \mathrm{s}^{-1}$, and at voltages varying between $+/-0.8 \mathrm{~V}$. Galvanostatic chargedischarge (GCD) involved cycling the supercapacitors between $0 \mathrm{~V}$ and $0.8 \mathrm{~V}$ with various current densities.

\section{Supporting Information}

Supporting Information is available from the Wiley Online Library or from the author.

All data supporting this study are openly available from the University of Southampton repository at https://doi.org/10.5258/SOTON/D0587

\section{Acknowledgements}




\section{WILEY-VCH}

The authors thank the EPSRC for supporting this research with grant reference EP/1005323/1 and $\mathrm{EP} / \mathrm{K} 031910 / 1$.

Received: ((will be filled in by the editorial staff))

Revised: ((will be filled in by the editorial staff))

Published online: ((will be filled in by the editorial staff)) 


\section{WILEY-VCH}

\section{References}

[1] W. Weng, P. Chen, S. S. He, X. M. Sun, H. S. Peng, Angew. Chem. Int. Ed. 2016, 55, 6140.

[2] T. Dias, Electronic Textiles Smart Fabrics and Wearable Technology, Woodhead Publishing, Sawston, Cambridge, UK, 2015.

[ 3 ] a) B. E. Conway, Electrochemical Supercapacitor Scientific Fundamentals and Technological Application, Kluwer Academic /Plenum Publishers, New York, US, 1999; b) X. L. Chen, R. Paul. L, M, Dai, National Science Review. 2017, 4, 3, 453; c) Z. Chen, International Materials Reviews. 2017, 62, 4, 173; d) A. González, E. Goikolea, J. A. Barrena, R. Mysyk, Renewable and Sustainable Energy Reviews. 2016, 58, 1189.

[4] U. Gulzar, S. Goriparti, E. Miele, T. Li, G. Maidcchi, A. Toma, F. Angelis, C. Capiglia and R. P. Zaccaria, J. Mater. Chem. A. 2016, 4, 16771.

[5] a) Z. Lu, Y. Chao, Y. Ge, J. Foroughi, Y. Zhao, C. Wang, H. Long and G. G. Wallace, Nanoscale. 2017, 9, 5063; b) F. M. Guo, R. Q. Xu, X. Cui,a L. Zhang, K. L. Wang, Y. W. Yao and J. Q. Wei, J. Mater. Chem. A. 2016, 4, 9311.

[6]W. Ma, S. Chen, S. Yang, W. Chen, W. Weng, and M. Zhu, ACS Appl. Mater. Interfaces. 2016, $8,14622$.

[7] a) H. Bashid, H. Ngee Lim, S.Kamaruzaman, S. Rashid, R. Yunus, N. Huang, C. Yin, M. Rahma, M. Altarawneh, Z. T. Jiang and P. Alagarsamy, Nanoscale Research Letters. 2017, 12, 


\section{WILEY-VCH}

246; b) Y. Chang, G. Han, Y. Xiao, H. Zhou, M. Li, D. Fu, W. Zhou, NEW CARBON MATERIALS. 2017, 32, 6, 581.

[8] M, Li. M. Zhu, J. Yu, H. Chen, Q. Li, small. 2017, 28, 1602994.

[9] S. Yong, J. Oven, M. Tudor, S. Beeby, Journal of Physics: Conference Series. 2015, 012074

[10] S. Yong, J. Oven, S. Beeby, Adv. Eng. Mater. 2018, 1700860.

[11] J. Wang, X. Li, Y. Zi, S. Wang, Z. Li, L. Zheng, F. Yi, S. Li, and Z. Wang, Adv. Mater. 2015, 27, 4830 .

[12] X. Pu, L. Li, M. Liu, C. Jiang, C. Du, Z. Zhao, W. Hu, Z. L. Wang, Adv. Mater. 2016, 28, $98-105$.

[13] K. Dong, Y. C. Wang, J. Deng, Y. J. Dai, S. L. Zhang, H. Y. Zou, B. H. Gu, B. Z. Sun and Z. L. Wang, ACS. Nano. 2017, 11(9), 9490.

[14] Z. Wen, M. H. Yeh, H. Y. Guo, J. Wang, Y. Zi, W. Xu, J. Deng, L. Zhu, X. Wang, C. Hu, L. Zhu, X. Sun, Z. L. Wang, Sci. Adv. 2016, 2, e1600097.

[15] Y. Song, J. Zhang, H. Guo, X. Chen, Z. Su, H. Chen, X. Cheng, H. Zhang, Appl. Phys. Lett. 2017, 111, 073901.

[16] S. Bauer, R. Gerhard-Multhaupt, G. Sessier, Physics Today. 2004, 57, 2, 37. 


\section{WILEY-VCH}

[17] J-J. Wang, T-H. Hsu, C-N. Yeh, J-W. Tsai and Y-C. Su, J. Micromech. Microeng. 2012, $22,015013$.

[18] J. Shi, D. Zhu, Z. Cao, S.P. Beeby, Journal of Physics: Conference Series. 2014, 660 (1), 012041.

[19 ]S. K. Ghosh, T. K. Sinha, B. Mahanty and D. Mandal, Energy technology. 2015, 3, 1190.

[20] W. Li, D. Torres, T. Wang, C. Wang and N. Sepulveda, Nano energy. 2016, 30, 649

[21] J. X. Zhu, F. Ma, H. Zhu, Energy technology. 2018, 6, 1112.

[22] S. Jie, S. Yong, S. Beeby, Smart Materials and Structures. 2018, 27, 084005 